\title{
Linking SOX10 to a slow-growth resistance phenotype
}

\author{
Cell Research (2014) 24:906-907. doi:10.1038/cr.2014.67; published online 23 May 2014
}

\begin{abstract}
Slow-cycling $B R A F^{V 600 E}$ melanoma cells are notoriously resistant to standard chemotherapy or targeted therapy but the underlying mechanism remains elusive. Now a new study unlocks this mystery and offers novel insights into developing more effective therapeutic interventions.
\end{abstract}

Molecular-guided targeted therapies have been effective in shrinking the bulk of BRAF ${ }^{V 600 E}$ tumors, particularly in patients with advanced or unresectable melanoma [1]. Unfortunately, therapeutic response is not durable, which is inevitably followed by tumor relapse. Thus, it is of great importance to elucidate the molecular basis that allows $B R A F^{V 600 E}$ melanoma cells to acquire resistance to targeted therapy and to potentially reveal new targets for combinatorial therapy. To date, numerous mechanisms have been identified as culprits that induce tumors to become refractory. The core non-genetic acquired resistance mechanisms include MAPK and PI3K pathways, which partially result from compensatory reactivation of receptor tyrosine kinases (RTKs) [2]. Among all RTKs, IGF-1R, PDGFR $\beta$, and ERBB3 have been identified to play critical roles in mediating acquired resistance [3-5].

Although harboring the $B R A F^{V 600 E}$ mutation, BRAF mutant colon cancers exhibit intrinsic resistance to BRAF inhibitor, vemurafenib [6]. This is attributed to the feedback activation of EGFR, another RTK. To test whether EGFR also mediates acquired resistance in BRAF mutant melanoma cells, Sun et al. [7] first examined paired pre- and post-treatment tumor biopsies of 16 melanoma patients. They found that the expression of EGFR was substantially increased in 6 out of 16 progressing tumor biopsies. Forced expression of EGFR retarded the proliferation of $B R A F^{V 600 E}$ melanoma cells, displaying certain features of oncogene-induced senescence (OIS). However, EGFR expression conferred an advantage for $B R A F^{V 600 E}$ melanoma cells to improve survival from MEK inhibition. To gain mechanistic insights into how EGFR is regulated in mediating acquired resistance, targeted shRNA screening was conducted in melanoma cells to focus on 661 chromatin modifiers. Prolonged exposure of A375 melanoma cells carrying the targeted shRNA library led to the selection and further expansion of cells resistant to vemurafenib. The resistant cells were further sorted by FACS to enrich EGFR ${ }^{\text {low }}$ cells, which represented $18.1 \%$ of the total resistant cells and were subjected to deep sequencing to identify enriched shRNAs. It should be noted that EGFR ${ }^{\text {high }}$ cells are not induced when treated with vemurafenib in the absence of targeted shRNA, suggesting that only the depletion of certain chromatin modifiers results in the acquired resistance that is mediated by EGFR. Deep sequencing then revealed that shRNA depleting SRY (sex determining region Y)-box 10 (SOX10) was enriched in resistant cells. The transcription factor, SOX10, is important for proper development of various neural crest-derived cell types including differentiated melanocytes. Knockdown of SOX10 led to an increase in EGFR expression by melanoma cells and a slow-growth phenotype. This is exemplified by the acquisition of hallmarks of OIS, which is consistent with a previous study. SOX $10^{\text {low }}$ senescent melanoma cells indeed showed resistance to vemurafenib. Sun et al. pinpointed that depletion of SOX10 led to activation of three TGF- $\beta$ signaling-dependent RTKs, which include EGFR, PDGFR $\beta$ and ERBB3. Expression of EGFR is decreased upon withdrawal of vemurafenib, suggesting that the EGFR-mediated resistance mechanism is reversible and adaptive. Because of the rapid decrease in EGFR expression following the removal of vemurafenib, EGFR ${ }^{\text {low }}$ melanoma cells may regain sensitivity to vemurafenib. This strategy may benefit a subset of $B R A F^{V 600 E}$ melanoma patients who have experienced a drug holiday.

Overall, this study has delineated the role of SOX10-TGF- $\beta$-EGFR/PDGFR $\beta /$ ERBB3 signaling axis in mediating adaptive and acquired resistance of $B R A F^{V 600 E}$ melanoma cells to vemurafenib. Targeting EGFR alone with EGFR inhibitor, Gefitinib, has not completely counteracted acquired resistance, implying the redundancy of multiple RTKs. However, combination of vemurafenib and PI3K inhibitor, GDC0941, was effective in inhibiting RTKs-mediated acquired resistance, underscoring the importance of targeting core acquired resistance mechanism including the PI3K pathway.

It is not entirely clear whether vemurafenib directly or indirectly suppresses SOX10 expression. The authors hypothesize that vemurafenib enriches a subpopulation of senescent SOX10 $10^{\text {low }}$ EGFR $^{\text {high }}$ melanoma cells which outgrow when the MAPK pathway is decreased to a certain threshold. This hypothesis resonates with progression of human 
senescent nevi into primary melanomas but warrants further investigation. It has been reported that vemurafenib by itself can induce cellular senescence in $B R A F^{V 600 E}$ melanoma cells [8]. It is not yet clear whether the SOX10-TGF- $\beta$ EGFR/PDGFR $\beta / E R B B 3$ signaling axis also plays a role in senescent cells.

Depletion of SOX10 led to the decrease in MITF expression, which is another important melanocyte lineage gene. However, MITF can also confer resistance to vemurafenib $[9,10]$. This shows that acquired resistance mechanisms are heterogeneous and suggests that distinct resistant subpopulations of SOX $10^{\text {high} / M_{T T F}}{ }^{\text {high }}$ and SOX $10^{\text {low }} /$ MITF $^{\text {low }}$ melanoma cells may co-exist.

The targeted shRNA library screening presented in this study is a vital approach to identifying genes that influence the adaptive pathway that will ultimately lead to acquired resistance. Targeting those genes early on will likely eradicate all malignant cells or at least delay the onset of acquired resistance, thus improving the efficacy of current targeted therapy approaches.

\section{Gao Zhang ${ }^{1,2}$, Meenhard Herlyn ${ }^{1,2}$}

${ }^{1}$ Molecular and Cellular Oncogenesis Program, ${ }^{2}$ Melanoma Research Center, The Wistar Institute, Philadelphia, PA 19104, USA

Correspondence: Meenhard Herlyn

E-mail: herlynm@wistar.org

\section{References}

1 Flaherty KT, Puzanov I, Kim KB, et al. $N$ Engl J Med 2010; 363:809-819.

2 Shi H, Hugo W, Kong X, et al. Cancer Dis- cov 2014; 4:80-93.

3 Villanueva J, Vultur A, Lee JT, et al. Cancer Cell 2010; 18:683-695.

4 Nazarian R, Shi H, Wang Q, et al. Nature 2010; 468:973-977.

5 Abel EV, Basile KJ, Kugel CH 3rd, et al. J Clin Invest 2013; 123:2155-2168.

6 Prahallad A, Sun C, Huang S, et al. Nature 2012; 483:100-103.

7 Sun C, Wang L, Huang S, et al. Nature 2014; 508:118-122.

8 Haferkamp S, Borst A, Adam C, et al. J Invest Dermatol 2013; 133:1601-1609.

9 Konieczkowski DJ, Johannessen CM, Abudayyeh O, et al. Cancer Discov 2014; 4:816-827.

10 Johannessen CM, Johnson LA, Piccioni F, et al. Nature 2013; 504:138-142. 